\title{
Using ANFIS to Predict Harmonic Distortion in Residential Building Loads: A case study in the Amazonian Region of Brazil
}

\author{
A. Albino Moisés Faro de Morais Junior ${ }^{1}$, B. Maria Emília de \\ Lima Tostes ${ }^{1}$, C. Ubiratan Holanda Bezerra ${ }^{1}$ and D. Thiago Mota Soares ${ }^{1}$ \\ ${ }^{1}$ Electrical Engineering Faculty, Federal University of Pará, Institute of Technology, Av. Augusto Corrêa n ${ }^{\circ} 01$, CEP \\ 66075-900, Belém, PA, Brazil \\ Phone/Fax number:+5591 981166285, e-mail: $\underline{\text { albinomorais@gmail.com, tostes@ufpa.br }}$
}

\begin{abstract}
With the increasing use of nonlinear loads in homes in Brazil comes the problem of harmonic injection in the power system and increasingly is a problem for the electric sector that needs to scale it. Knowing the loads that consume energy and inject harmonics into the system is important so that solutions are sought to make the use of the system more efficient and improve the quality of the energy that circulates in the electrical grid. This work presents simulations of DHTv and DHTi of a set of residences in order to predict the behaviour of the load over time, using previous measurements. The modelling is conducted using an ANFIS, which uses a neural network to adjust the parameters of the output that uses fuzzy rule to determine the output values of the system.
\end{abstract}

\section{Key words}

Electric power quality; Modelling; harmonic distortion; neuro-fuzzy system, ANFIS

\section{Introduction}

The technological advances of the last decades have led to major developments in the electronics sector, which have been passed on to new consumer products, thus benefiting an increasing number of consumers, who are having access to new products that are better suited prices and introducing new functions.

In addition to this, there is a strong call for sustainability that encourages the use of efficient equipment to reduce consumption, such as the use of compact bulbs and LED bulbs with electronic ballasts. This has also contributed to the degradation of voltage and current waveforms in residential buildings. Today the low voltage consumers of a power distribution system are injecting harmonics into the distribution network at a level that already deserves special concerns and requires studies to better assess the impacts that these distributed sources of harmonic generation can have on quality of electricity.

Given this scenario, the Brazilian Electricity Regulatory Agency (ANEEL) has developed specific regulation that establishes procedures for electric utilities on the quality of electric power, similar to other international standards such as IEEE-519 [1] and IEC-61000-3- [2].

From the module 8 of PRODIST (Procedures for Distribution of Electric Energy in the Brazilian National Electric System) [3] set forth by ANEEL that establishes the procedures related to electric power quality (QEE), performance indicators were created and acceptable limits set for these indicators in order to assess the energy quality of a given point in the system.

Considering this constant growth of non-linear loads in distribution networks, electric power distribution companies need to look for tools to assess behaviour and predict what may happen to the system to prevent an event from destabilizing or even shutting down the distribution network.

Studies show that designing prediction models for harmonic distortion becomes difficult, since factors such as load variations interfere in its precision response [4]

Among many works [5-11], the determination of dominant harmony at source is the main focus, rather than determining the individual harmonic contribution. Initially, the determination of the dominant harmonic source was addressed by harmonic-based techniques [8-10], and other methods based on harmonic impedance determination [57]. Some other methods have been proposed for estimating the harmonic contributions of the utility and the client in voltage in the CCP [12-14]. In these methods, the focus is mainly on separating the harmonic contribution of a customer from that of the supply system in the CCP. Unsar et al. $[15,16]$ use time-synchronized field measurements of an iron and steel plant to identify the contribution of the current harmonics in the $\mathrm{CCP}$, and within the industrial Plant. In general, these approaches seek to determine the proportion of harmonic distortion generated by the load itself and by what is in the power grid. 
Unlike the approaches that consider CCP as the point of interest, the authors in [17-20] developed data-based methodologies to determine the contribution of harmonic distortion of individual loads at a specific location of the power grid, not requiring the $\mathrm{CCP}$, using linear correlation analysis.

One of the tools that can help understand and better plan the actions for maintenance of the distribution system is the prediction of load on the distribution transformers for harmonic distortions. This will allow load flow studies on the main harmonic frequencies of interest to be carried out in order to evaluate the harmonic impacts on the distribution network and to plan the future operation of the system more efficiently, economically and safely.

Predicting load on distribution transformers is a difficult task today, mainly due to the lack of data from distribution networks. This work aims at the development of a tool using artificial intelligence that will predict load on the harmonic frequencies in transformers in the distribution network.

The great advantage of this developed tool is the ability to model more precise patterns using a prediction of harmonic states of the loads connected in the low voltage that helps in the creation of pseudo-actions for distribution networks, where real measurements are difficult and costly.

First, we will present the data that consisted of measurements in distribution transformers in order to acquire data for the design of a neuro-fuzzy system, which was the computational tool chosen to predict the load intelligently. Because it is a universal nonlinear approximator and because it has the learning ability of a neural network and the use of the human knowledge of a Fuzzy system, this technique is suitable for the modelling of systems with imprecisions and that did not have formal models for the representation .

The purpose of this article is to implement a computational system that predicts harmonic distortion in energy distribution transformers in order to provide an approximate description of the behaviour of transformers in the distribution network at different times of the day, week and year.

The computational structure used makes feeder currents prediction of a set of upper middle class residential homes on typical days such as a normal weekday and on weekends. For this, values are used as inputs that characterize the date and time for the prediction. This information is important because it is known that the load profile strongly depends on the time and day of the week, since the daily and weekly cycles have relative repetitiveness.

\section{NEURO-FUZZY SYSTEM}

The computational tool adopted for predicting fundamental and harmonic loads was the Fuzzy system. However, to work with this artificial intelligence tool, a set of rules is required to relate the input to the output. This is often a highly exhaustive and time-consuming task, since it is necessary for the rules to provide for all inputs and possible outputs, as well as the concern to eliminate any kind of conflict in the THEN part of the rules, which would lead to two different outputs for same input.

To solve this problem, this paper proposes the use of a neuro-fuzzy network, where the network will be trained using neural networks techniques to assemble the rules that will be later used in the fuzzy system assembled from the coefficients obtained in the network training. In this case, the purpose of the neural network is to process the information according to its previous training with input and output data. The choice of the neural network is because the neural networks are extremely parallel, since their numerous operations are executed simultaneously.

The chosen neuro-fuzzy system was the system created by J. S Roger Jang called ANFIS (Adaptive Network-based Fuzzy Inference System) that makes an adaptation of the input and output values to a rule base that interconnects all inputs and outputs, thus forming a robust rule base that creates a fuzzy inference system that contemplates all possible inputs.

ANFIS was chosen because it is used for prediction and function approximation applications. In addition, it is a Takagi-Sugeno system and it is shows that its first-order model is a universal approximation. Its popularity is so great that it has been implemented in MatLab. An example of a first-order Takagi-Sugeno system can be seen below.

Having two input variables $\mathrm{x}$ and $\mathrm{y}$ and one output $\mathrm{z}$ and the rules (1) and (2):

Rule 1: IF $x$ is $A_{1}$ and $y$ is $B_{1}$ THEN

$f_{1}=p_{1} \times x+q_{1} \times y+r_{1}$

Rule 2: IF $x$ is $A_{2}$ and $y$ is $B_{2}$ THEN

$f_{2}=p_{2} \times x+q_{2} \times y+r_{2}$

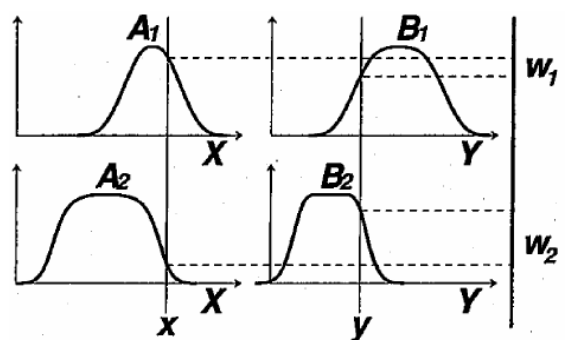

Fig. 1. Example of the first-order Takagi-Sugeno system Thus in Figure 1 we have (3):

$Z=\frac{w_{1} \times f_{1}+w_{2} \times f_{2}}{w_{1}+w_{2}}$

Figure 2 shows the architecture of the ANFIS model and its several layers. 


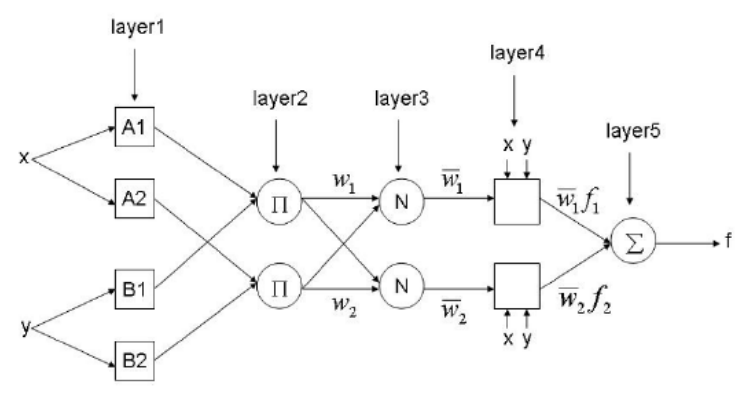

Fig. 2. Architecture of the ANFIS System

\section{A. Layer Description:}

Layer 1: Calculates the degree of relevance with which the inputs satisfy the values or linguistic terms associated with these nodes. It is called background layer.

Layer 2: Each node of this layer corresponds to a rule and calculates to what degree the consequence of the rule is being met.

Layer 3: This layer normalizes the values of the previous layer.

Layer 4: In this layer, the outputs of the neurons are calculated by the product of the normalized values in the previous layer and the values of the consequent ones of the rule. It is called the consequent layer.

Layer 5: The node of this last layer of the architecture calculates the precise output of the system and, together with the nodes of layers 3 and 4, promotes defuzzification.

This system uses Fuzzy-grid Adaptive Partitioning. Its learning (identification of the structure and parameters) is done in two steps that repeat until the criterion of stop is reached.

\section{B. Description of steps:}

Step 1: The parameters of the antecedents are fixed and the consequent ones are adjusted by the MQD method estimation by Ordinary Least Squares (OLS).

Step 2: The consequent parameters are fixed and the antecedents are adjusted by the Descending Gradient algorithm.

\section{IMPLEMENTATION}

In the data implementation phase, they were collected from a distribution transformer that fed middle class residential units from the northern region of Brazil, specifically in the state of Amazonas.

Measurements were made in the morning, afternoon and evening periods for weekdays and weekends, in order to reproduce the load profile at any desired period after the development and implementation of the computational tool.

A fundamental and/or harmonic load prediction system will be implemented, which will have as input the day of the week and the time of day in which the data were obtained. The output will be composed by the fundamental currents and/or harmonics in the three phases, which characterize the conditions of load of the transformers, in the respective frequencies.

\section{A. Data collection for network learning}

The characterization of the distributed sources of generation of harmonics in the feeder was done by a measurement plan that was conducted during several days.

Measurements were made to build a sampling of the harmonic injection of the transformer, by recording the voltage and current waveforms on the transformers every ten minutes on common weekdays and weekend days at times including the morning, afternoon and evening periods.

Measurements were conducted with a PowerVisa, a power quality analyser from Dranetz-BMI, a three-phase model, which can collect data for days and even entire weeks without the need to remove data from them. The power quality analyser used has nominal Cat $600 \mathrm{~V}$ capacities. This instrument makes harmonic decomposition up to the 63rd order, expressing this spectral decomposition through bar graphs.

To validate the developed model, a measurement database was used on different days, so that training and validation of the output data of the neuro-fuzzy network were done in order to show a clear result that ANFIS has a high learning power and serves perfectly for the analysis and prediction of fundamental and harmonic load of the transformer she trained.

\section{B. Methodology}

The databases used for modelling were collected for weeks on end, allowing for the possibility of having several measurements available for the same day of the week, which makes the system more reliable, since with a large range of data one can discard the measurements which presented an atypical shape due to abrupt variations of the network and even voltage drop.

The data collection process is done every second, and the equipment was programmed to store this data every 10 minutes, and then the various values obtained during 10 minutes are filled in for the storage of only one value. The obtained file is transferred to the computer and using the program provided by the power quality analyser manufacturer, and converted to text.

A fundamental and harmonic load prediction system DHTi\% - was implemented using the tool called MATLAB ANFIS, which will have previous known data as input and the output will be composed by DHTi\%.

The first step in implementing the system for load prediction is the preparation of the input data. In this case, the input data is in files with the extension ".dat". It is worth mentioning that the ANFIS system available in MatLab allows several entries, but only one output. The preparation of these files was done using the Microsoft Excel spreadsheet.

Because it is a time-series prediction, we need to use known time-series values to the point in time, say $\mathrm{t}$, to predict the 
value at some point in the future, say, $\mathrm{t}+\mathrm{P}$. The standard method for this type of prediction is to create a mapping of 4 sample points, sampled at each $\Delta$ units at time, [x ( $\mathrm{t}-3), \mathrm{x}$ $(\mathrm{t}-2), \mathrm{x}(\mathrm{t}-1), \mathrm{x}(\mathrm{t})]$, for a predicted future value $\mathrm{x}(\mathrm{t}+1)$. Following conventional configurations to predict time series, input training data for ANFIS is a four-dimensional vector as follows:

[x ( t-3), x (t-2), x (t-1), x (t), x ( t + 1)]

Where $[\mathrm{x}(\mathrm{t}-3), \mathrm{x}(\mathrm{t}-2), \mathrm{x}(\mathrm{t}-1), \mathrm{x}(\mathrm{t})]$ will be used as input $\mathrm{y}$ $[x(t+1)]$ which will be the output of the ANFIS network.

After the conversion, the data is loaded into MATLAB's ANFIS, through a routine that works to prepare the input and output matrix of data. As the results obtained varied with the number of functions of inferences of the layers, but did not necessarily improve with the increase of this number of functions, we chose to create a routine that varies the number of functions of inference of the layers of input 2 . This variation occurred independently for each layer and ranged from 2 to 4 inference functions for each input. With this we can compare the results and present the best answer for each case.

We also tested the six types of pertinence functions accepted by the MATLAB ANFIS routine, namely dsigmf (composed of the difference between two Sigmoids), gaussmf (Gaussian), gauss $2 \mathrm{mf}$ (Combination between two Gaussian ones), gbellmf (form of $\pi$ ) and psigmf (Product between two Sigmoidals) in order to determine the best function for each case. It was noticed that there is no function that stands out and is the best for all cases, and it is necessary to vary this input data for the creation of the initial neural network.

The ANFIS network is trained until it satisfies a stopping criterion that can be the number of epochs or the value of the error, whichever is reached first by the ANFIS.

In order to arrive at a satisfactory result in the load prediction of this work, several tests were needed as we noticed the existence of a saturation point for the reduction of the error, which depends directly on the number of rules requested. Then, in order for the minimum value of the error to be reached, several tests were performed until a number of adequate training rules reached the minimum value of the mean error.

Each DHTi predicted in this work had a number of rules that met the criterion of the minimum error, since we tried to maintain a commitment to the optimization of the system of load prediction.

To validate this work, the results obtained for phase A DHTi on a weekday (Monday) and phase B DHTi on a weekend day (Saturday) will be shown.

\section{RESULTS}

\section{A. DHTi Prediction for Monday's Phase A}

The DHTi prediction model on the transformer was constructed based on data from the following one-day load curves on Monday, where data from five Mondays were used in full, with half of the data being used for training and the other half for testing and validation.

The results are presented in graphical form, made of three graphs. In the first one, the behaviour of the Mean Root Quadratic Error for the training and test data is presented.

In the second graph is shown the modelling of the ANFIS system, where the blue asterisks represent the actual output that is known and the green circles are the ANFIS predictions.

The third graph shows the prediction error of the ANFIS output for the values tested.

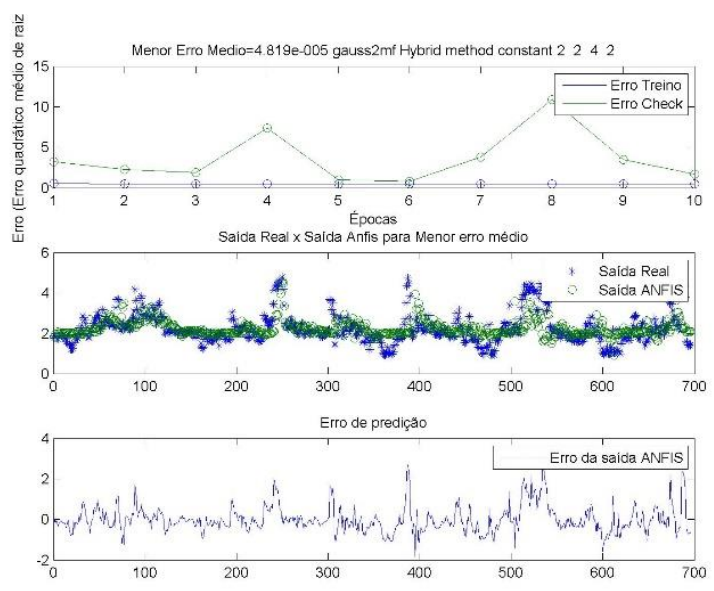

Fig. 3. DHTi Prediction for Monday's Phase A

The best result for the tested ANFIS networks was obtained for the type of inference function combination of two Gaussian functions, for the Hybrid optimization method, type of function of constant output inference and with the number of functions of 2-2- 4-2, which represents the number of inference functions of each of the four inputs.

We noticed that the model presents a good result, having most of the answers of the ANFIS coinciding with the real answers of the system. It is worth mentioning that this was the best among the several results presented by the MATLAB routine.

\section{B. DHTi prediction for Phase B on Saturday}

The DHTi prediction model on the transformer was constructed based on data from the following one-day load curves on Saturday, where data from five Saturdays were used in full, with half of the data being used for training and the other half for testing and validation.

The results are presented in graphical form, made of three graphs. In the first one, the behaviour of the Mean Root Quadratic Error for the training and test data is presented.

In the second graph is shown the modelling of the ANFIS system, where the blue asterisks represent the actual output that is known and the green circles are the ANFIS predictions. 
The third graph shows the prediction error of the ANFIS output for the values tested.

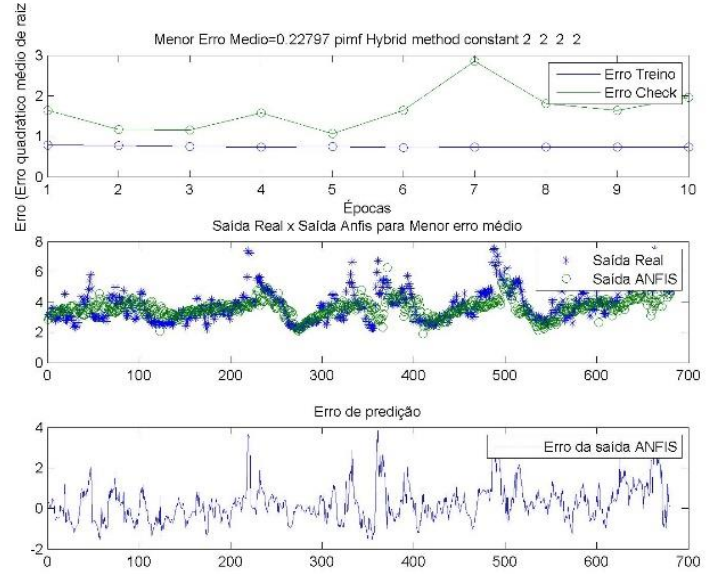

Fig. 4. DHTi prediction for Phase B on Saturday

In the same way, this result contributes to the acceptance of this model, since it discards the possibility of coincidence and presents consistent data, as the results obtained when they are not coincident are very close to the real results.

\section{DHTv prediction for Monday's Phase A}

The DHTv prediction model on the transformer was constructed based on data from the following one-day load curves on Monday, where data from five Mondays were used in full, with half of the data being used for training and the other half for testing and validation.

The results are presented in graphical form, made of three graphs. In the first one, the behaviour of the Mean Root Quadratic Error for the training and test data is presented.

In the second graph is shown the modelling of the ANFIS system, where the blue asterisks represent the actual output that is known and the green circles are the ANFIS predictions.

The third graph shows the prediction error of the ANFIS output for the values tested.

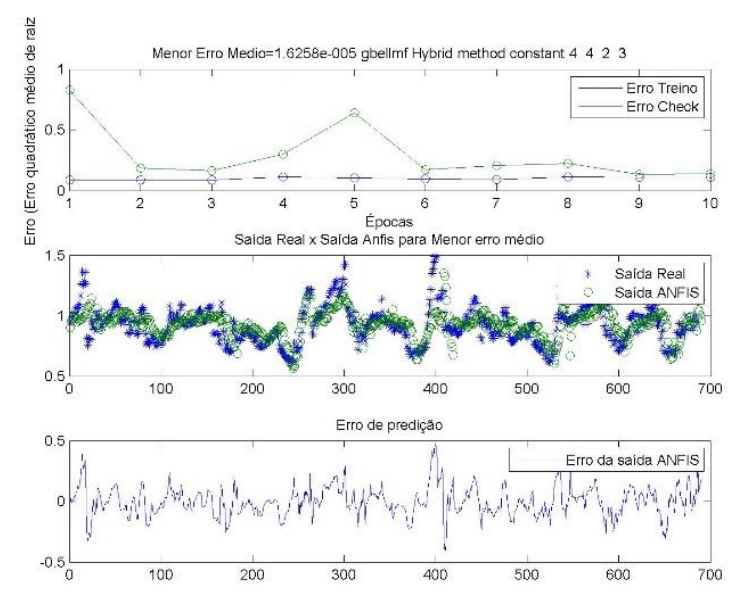

Fig. 5. DHTv Prediction for Monday's Phase A
The results show that the tool is also a good harmonic state estimator of the DHTv, because when the values of the ANFIS output do not match the actual values, they come close to reality.

\section{DHTv prediction for Phase C of Sunday}

The DHTv prediction model on the transformer was constructed based on data from the following one-day load curves on Sunday, where data from five Sundays were used in full, with half of the data being used for training and the other half for testing and validation.

The results are presented in graphical form, made of three graphs. In the first one, the behaviour of the Mean Root Quadratic Error for the training and test data is presented.

In the second graph is shown the modelling of the ANFIS system, where the blue asterisks represent the actual output that is known and the green circles are the ANFIS predictions.

The third graph shows the prediction error of the ANFIS output for the values tested.

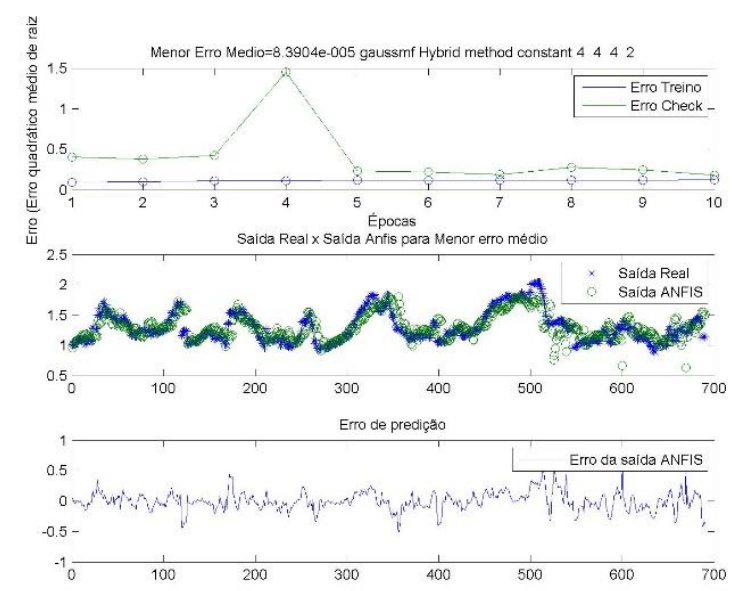

Fig. 6. DHTv prediction for Phase B on Sunday

It is evident that even though the values are not identical, the ANFIS successfully predicts the behaviour of DHTV even for non-trained values.

\section{CONCLUSION}

This article presents the implementation of a computational system, using ANFIS Neuro-fuzzy network, which makes predictions through the Total Harmonic Distortion of Percent Current (DHTi\%) - trying to describe the approximate behaviour of the transformers of the distribution network, in the different periods of days and days of the week.

For the DHTi\%, it is observed that the model developed in most of the predicted points coincided with the real points. Therefore, the ANFIS is a good tool to use in the prediction of total harmonic distortion in transformers.

In all cases implemented, the network response came very close to the actual response. Even for situations where the 
ANFIS does not present a response that is so close to reality, it follows the form of expected output.

For presenting promising results, this work could be used in future studies of harmonic load flow for distribution networks, with the great advantage of being able to model more precise patterns using a prediction of harmonic states of loads connected to low voltage, which helps in the creation of pseudo-measures for distribution networks, where real measurements are difficult and costly to obtain.

\section{References}

[1] IEEE Std 519-1992 - IEEE recommended practices and requirements for harmonic control in electric power systems. Institute of Electrical and Electronics Engineers, Inc

[2] IEC 61000-3-6 - Electromagnetic compatibility (EMC) - part 3-6: limits - assessment of emission limits for the connection of distorting installations to MV, HV and EHV power systems. Edition 2.0. 2008

[3] ANEEL Módulo 8 - Procedimentos de Distribuição de Energia Elétricano Sistema Elétrico Nacional - PRODIST. 2007.

[4] MANITO, A. R. A.; BEZERRA, U. H.; EMÍlIA, M.; TOSTES, D. L.; SOARES, T. M. Estimação da Contribuição de Cargas Não Lineares na Distorção Harmônica de Tensão de um Barramento de Interesse do Sistema Elétrico Utilizando Rede Neural Artificial. Simpósio Brasileiro de Sistemas Elétricos - SBSE, Foz do Iguaçu PR, 2014

[5] Santos, I.N., Oliveira, J.C.: 'Modified superposition method for assignment of responsibilities on harmonic distortion'. Proc. of 2011 11th Int. Conf. on Electrical Power Quality and Utilization, 2011, pp. 1-5

[6] de Andrade, G.V. Jr., Naidu, S.R., Neri, M.G.G., et al.: 'Estimation of the utility's and consumer's contribution to harmonic distortion', IEEE Trans. Instrum. Meas., 2009, 58, (11), pp. 3817-3823

[7] Li, C., Xu, W., Tayjasanant, T.: 'A 'critical impedance'-based method for identifying harmonic sources', IEEE Trans. Power Deliv., 2004, 19, (2), pp. 671-678

[8] Xu, W., Liu, X., Liu, Y.: 'An investigation on the validity of powerdirection method for harmonic source determination', IEEE Trans. Power Deliv., 2003, 18, (1), pp. 214-219

[9] Xu, W., Liu, Y.: 'A method for determining customer and utility harmonic contributions at the point of common coupling', IEEE Trans. Power Deliv., 2000, 15, (2), pp. 804-811

[10] Swart, P.H.: 'On techniques for localization of sources producing distortion in three-phase network', Eur. Trans. Electr. Power Eng., 1996, 6, (6), pp. 391-396
[11] Cristaldi, L.: 'Harmonic power flow analysis for the measurement of the electric power quality’, IEEE Trans. Instrum. Meas., 1995, 44, (3), pp. 683-685

[12] Pfajfar, T., Blazic, B., Papic, I.: 'Harmonic contributions evaluation with the harmonic current vector method', IEEE Trans. Power Deliv., 2008, 23, (1), pp. 425-433

[13] Nino, E.E., Xu, W.: 'Measurement of harmonic sources in three-wire single-phase supply systems', IEEE Trans. Power Deliv., 2007, 22, (4), pp. 2527-2533

[14] Davis, E.J., Emanuel, A.E., Pileggi, D.J.: 'Harmonic pollution metering: theoretical considerations', IEEE Trans. Power Deliv., 2000, 15, (1), pp. 19-23

[15] Unsar, O., Salor, O., Cadirci, I., et al.: 'Identification of harmonic current contributions of iron and steel plants based on timesynchronized field measurements - part I: at PCC', IEEE Trans. Ind. Appl., 2014, 50, (6), pp. 4336-4347

[16] Unsar, O., Salor, O., Cadirci, I., et al.: 'Identification of harmonic current contributions of iron and steel plants based on timesynchronized field measurements - part II: inside plants', IEEE Trans. Ind. Appl., 2014, 50, (6), pp. 4348-4355

[17] Yin, Z., Sun, Y., YU, T.: 'New methods exploration for harmonic source identification technologies'. Fourth Int. Conf. on Electric Utility Deregulation and Restructuring and Power Technologies (DRPT), July 2011, pp. 399-402

[18] Mazin, H.E., Xu, W., Huang, B.: 'Determining the harmonic impacts of multiple harmonic-producing loads', IEEE Trans. Power Deliv., 2011, 26, (2), pp. 1187-1195

[19] Farhoodnea, M., Mohamed, A., Shareef, H., et al.: 'An improved method for determining contribution of utility and customer harmonic distortions in a power distribution system', Int. J. Electr. Eng. Inf., 2010, 2, (3), pp. 204-215

[20] CIGRE Working Group 36.05/CIRED2: 'Review of methods for measurement and evaluation of the harmonic emission level from an individual distorting load'. WGCC02, January 1999

[21] SHAW, Ian S.; SIMÕES, Marcelo Godoy. Controle e Modelagem FUZZY. Ed. Edgard Blücher LTDA. $1^{\mathrm{a}}$ Edição.

[22] Fuzzy Logic Toolbox User's Guide, 1998 by The MathWorks, Inc.

[23] Li-Xin Wang, A Course in Fuzzy Systems and Control, 1997 by Prentice Hall, Inc.

[24] Tostes, M. E. L.; “Avaliação de Impactos na Rede de Distribuição Causados pela geração de Harmônicos em Consumidores em baixa Tensão"; Tese de Doutorado Defendida em Dezembro de 2003, Curso de pós - Graduação em Engenharia Elétrica da Universidade Federal do Pará 